\title{
artigo
}

Santos, M.M.D.; Peters, A.A.; Figueiredo, M.A.G.; Carreiro, M.A.; Magalhães, M.C.; Araújo, E.P.;

Controle glicêmico, suporte social percebido e o autocuidado de indivíduos com diabetes tipo 2

\section{Controle glicêmico, suporte social percebido e 0 autocuidado de indivíduos com diabetes tipo 2}

\author{
Glycemic control, perceived social support and self-care of individuals with type 2 diabetes \\ Control glicémico, apoyo social percibido y cuidado personal de personas con diabetes tipo 2
}

\begin{abstract}
RESUMO
Objetivo: Avaliar o grau de suporte social percebido e sua relação com o autocuidado e o controle glicêmico dos indivíduos diabéticos tipo 2. Metodologia: Pesquisa transversal, realizada com 154 indivíduos diabéticos com idade superior a 18 anos, acompanhados em unidades de saúde. Foram analisadas variáveis sociodemográficas, antropométricas, clínicas, de autocuidado e de suporte social relacionadas ao controle glicêmico. Para a análise das variáveis de caráter genérico, foi realizado um estudo de frequência simples e calculadas as medidas de tendência central. Para verificar a associação das comorbidades com o controle glicêmico foi utilizado o teste Qui-quadrado. Resultados: Os participantes atingiram boa pontuação em algumas atividades do autocuidado e, apesar de terem pouco apoio social, se sentiam satisfeitos. Ao se associar as atividades de autocuidado com o suporte social recebido, não houve associação estatisticamente significativa. Conclusão: Houve associação entre algumas comorbidades, como Hipertensão, obesidade, nefropatias, depressão e o uso de insulina com o controle glicêmico, o que reforça a necessidade de estimular cada vez mais a busca do controle entre os indivíduos com diabetes tipo 2.
\end{abstract}

DESCRITORES: Diabetes Mellitus; Apoio Social; Autocuidado; Enfermagem; Glicemia; Doenças Metabólicas.

\section{ABSTRACT}

Objective: To evaluate the degree of perceived social support and its relationship with self-care and glycemic control of type 2 diabetic individuals. Methodology: Cross-sectional research, carried out with 154 diabetic individuals over the age of 18, monitored in health units. Sociodemographic, anthropometric, clinical, self-care and social support variables related to glycemic control were analyzed. For the analysis of generic variables, a simple frequency study was carried out and the measures of central tendency were calculated. To check the association of comorbidities with glycemic control, the Chi-square test was used. Results: Participants achieved good scores in some self-care activities and, despite having little social support, they were satisfied. When associating self-care activities with the social support received, there was no statistically significant association. Conclusion: There was an association between some comorbidities, such as hypertension, obesity, kidney disease, depression and the use of insulin with glycemic control, which reinforces the need to increasingly encourage the search for control among individuals with type 2 diabetes.

DESCRIPTORS: Diabetes Mellitus; Social Support; Self Care; Nursing; Blood Glucose; Metabolic Diseases.

\section{RESUMEN}

Objetivo: Evaluar el grado de apoyo social percibido y su relación con el autocuidado y el control glucémico de los individuos con diabetes tipo 2. Metodología: Investigación transversal, realizada con 154 individuos con diabetes mayores de 18 años, monitoreados en unidades de salud. Se analizaron variables sociodemográficas, antropométricas, clínicas, de autocuidado y de apoyo social relacionadas con el control glucémico. Para el análisis de variables genéricas, se realizó un estudio de frecuencia simple y se calcularon las medidas de tendencia central. Para verificar la asociación de comorbilidades con el control glucémico, se utilizó la prueba de Chi-cuadrado. Resultados: Los participantes lograron buenos puntajes en algunas actividades de autocuidado y, a pesar de tener poco apoyo social, quedaron satisfechos. Al asociar las actividades de autocuidado con el apoyo social recibido, no hubo asociación estadísticamente significativa. Conclusión: hubo una asociación entre algunas comorbilidades, como hipertensión, obesidad, enfermedad renal, depresión y el uso de insulina con control glucémico, lo que refuerza la necesidad de alentar cada vez más la búsqueda de control entre las personas con diabetes tipo 2.

DESCRIPTORES: Diabetes Mellitus; Apoyo Social; Autocuidado; Enfermería; Glucemia; Enfermedades Metabólicas.

RECEBIDO EM: 19/03/2020 APROVADO EM: 20/03/2020 


\section{Margarida Maria Donato dos Santos}

Enfermeira. Doutora em Ciências da Saúde pela Unicamp. SP, Brasil.

ORCID: 0000-0001-8681-5582

\section{Angela Aparecida Peters}

Enfermeira. Doutoranda pela Escola de Enfermagem Anna Nery da Universidade Federal do Rio de Janeiro. RJ, Brasil. ORCID:0000-0002-0363-8371

\section{Mariangela Aparecida Gonçalves Figueiredo}

Enfermeira. Doutora em História da Enfermagem pela Escola de Enfermagem Anna Nery da Universidade Federal do Rio de Janeiro. RJ, Brasil.

ORCID: 0000-0003-1382-7819

\section{Monica de Almeida Carreiro}

Enfermeira. Doutora em Enfermagem pela Universidade Federal do Rio de Janeiro. RJ, Brasil. ORCID: 0000-0003-1594-6491

\section{Maria da Consolação Magalhães}

Enfermeira. Doutora em Saúde pela Universidade Federal de Juiz de Fora. Enfermeira do Hospital Universitário da UFJF. MG, Brasil.

ORCID: 0000-0002-1480-7046

\section{Eliana Pereira de Araújo}

Enfermeira. Doutora. Professora Associada da Faculdade de Enfermagem Unicamp. SP, Brasil. ORCID: 0000-0002-7539-8477

\section{INTRODUÇÃO}

0 diabetes é uma doença de rápido crescimento entre as doenças crônicas. Integra um grupo de doenças metabólicas caracterizadas por níveis elevados de glicose no sangue. Suas complicações determinam uma carga enorme de cuidados de saúde, com muitos desafios para indivíduos e sistemas de saúde ${ }^{(1)}$.

Uma multiplicidade de fatores está envolvida na epidemia global do diabetes mellitus (DM), entre eles: o aumento da obesidade, o sedentarismo, a introdução de dietas ocidentalizadas baseadas no fast-food, desagregação do apoio da família, além do estresse resultante de longas horas de trabalho, da concorrência profissional intensa, todos resultando em falta de tempo para o exercício físico e uma negligência geral da saúde pessoal ${ }^{(1)}$.

No Brasil, a prevalência de DM na população adulta na década de 1980 foi estimada em 7,6\% ${ }^{(2)}$. Atualmente, existem estudos indicando taxas mais elevadas como $13,5 \%$ em São Carlos - $S P^{(3,4)}$ e $15 \%$ em Ribeirão Preto - $\mathrm{SP}^{(4)}$. O Estudo Longitudinal de Saúde do Adulto - ELSA ${ }^{(5)}$, um estudo multicêntrico sobre diabetes, realizado com 18.000 indivíduos de seis instituições de ensino superior, iniciado em 2008, revelou que $19 \%$ dos participantes eram diabéticos e destes, $53 \%$ não tinham diagnóstico prévio.

Estudos afirmam que vários países têm se preocupado com as pesquisas relacionadas com saúde e sua relação com o suporte social. Porém, no que diz respeito à necessidade de suporte social entre as pessoas com doenças crônicas, em especial o DM, pouca atenção tem sido dada em países como Bra$\mathrm{sil}^{(6)}$. Aliado a isso, existem poucas publicações da enfermagem relacionadas ao suporte social às pessoas com diabetes. Este fato dificulta o cuidado aos diabéticos, pois ele é uma forma de auxílio aos indivíduos em suas necessidades emocionais e de assistência à saúde, de modo que se sintam seguros, amados e cuidados, com sensação de bem-estar, favorecendo o desenvolvimento das atividades de autocuidado.

Barrera $^{(7)}$ conceitua o suporte social percebido como a avaliação cognitiva que $o$ indivíduo faz em relação aos vários domínios da sua vida, na qual ele se vê seguramente conectado a outros usuários e se sente ama- do e estimado pelos outros. É importante que seja feita a distinção entre apoio social e rede social, pois, apesar de interligados, possuem diferenças entre si. A rede social se refere à dimensão estrutural ou institucional ligada a um indivíduo, exemplificada por rede da vizinhança, as organizações religiosas, o sistema de saúde e o escolar ${ }^{(8)}$.

A meta no tratamento ao indivíduo com diabetes do tipo 2 (DM2) é levá-lo a atingir um bom controle glicêmico para a prevenção de complicações relacionadas à doença $\mathrm{e}$, portanto, o autocuidado deve ser estimulado. $\mathrm{O}$ autocuidado e o apoio de pessoas do convívio com o diabético, como familiares, amigos, vizinhos, pessoas da comunidade, incluindo a equipe de saúde, são fatores importantes na ajuda ao indivíduo para o alcance do seu controle glicêmico.

O problema da dificuldade do controle glicêmico em longo prazo é um tema de grande interesse para o diabético, já que está suficientemente demonstrado que a única maneira eficaz de prevenir os transtornos degenerativos próprios de diabetes é um controle estrito da glicemia, evitando flutuações importantes ${ }^{(9)}$. Apesar de existir no Brasil um programa ministerial 
para atendimento ao diabético na atenção primária e este possuir diretrizes e ações sistematizadas para serem aplicadas pelos profissionais de saúde aos diabéticos, observa-se na prática que estes indivíduos possuem dificuldades de atingir um bom controle glicêmico.

Como modo de prevenir e tratar doenças crônicas, dentre elas o DM, a Organização Mundial de Saúde (OMS) orienta a educação para o autocuidado, que visa permitir o envolvimento da pessoa em seu tratamento e ao esquema terapêutico proposto $^{(10)}$. O autocuidado descreve e explica a prática de cuidados executados pela pessoa portadora de uma necessidade para manter a sua saúde e o seu bem-estar. Envolve a participação do indivíduo com autonomia, liberdade e responsabilidade nas escolhas que objetivam seu bem-estar, tornando-se necessário que seja capaz de fazê-las. Significa deixar de ser passivo em relação aos próprios conceitos de autocuidado e diretrizes médicas.

O suporte social pode ser definido como o grau com que relações interpessoais atendem a determinadas necessidades, ou como "uma troca de recursos entre (pelo menos) duas pessoas, que visam aumentar o bem-estar do receptor" ${ }^{\text {(11) }}$, ou seja, o suporte emocional ou prático dado pela família e/ou amigos na forma de afeto, companhia, assistência e informação, tudo que faz o indivíduo ser amado, estimado, cuidado, valorizado e seguro ${ }^{(12)}$.

A educação em saúde com foco no autocuidado entre diabéticos tipo 2 e a organização de grupos de suporte podem ser consideradas estratégias para o enfrentamento da doença pelos indivíduos, família, comunidade e profissionais de saúde. Conhecer e avaliar o suporte social dos indivíduos com DM2 e o autocuidado desempenhado por eles é muito importante para o planejamento de intervenções específicas por parte dos enfermeiros para ajudar na adaptação às mudanças impostas pelo tratamento ${ }^{(6)}$.

Diante deste contexto, este estudo teve o propósito de avaliar o grau de suporte social percebido e sua relação com o autocuidado e o controle glicêmico dos indivíduos diabéticos tipo 2 .

\section{METODOLOGIA}

Trata-se de um estudo transversal de abordagem quantitativa. A pesquisa foi realizada em quatro unidades de Atenção Primária à Saúde de uma cidade do interior de Minas Gerais, no período compreendido entre 2015 e 2016, com indivíduos com DM2. Foram incluídos no estudo os indivíduos com DM2 cadastrados nas unidades, com idade mínima de 18 anos e capazes de responder verbalmente; e excluídos os diabéticos com dificuldade visual que os impedisse de desenvolver as atividades da vida diária ,como ler o nome dos medicamentos e auto aplicar-se insulina, além dos que tivessem referido o uso de álcool com mais de cinco doses (maior ou igual a $30 \mathrm{~g} / \mathrm{dia}$ para homens e $15 \mathrm{~g} /$ dia mulheres) e uso de drogas ilícitas, pois estes fatores sugerem alterações em algumas das variáveis investigadas. A amostra foi por conveniência, constituída por 154 indivíduos de ambos os sexos com diagnóstico médico de DM2 que compareceram à unidade para consulta ou outro procedimento de saúde e determinada após o teste piloto.

Foram consideradas neste estudo as variáveis sociodemográficas e clínicas, como Hipertensão Arterial, obesidade, doenças osteoarticulares, hipotireoidismo, a retinopatia e a neuropatia. E também as atividades do autocuidado, descritas no questionário de atividades do autocuidado e as perguntas do questionário do suporte social. A variável padrão ouro e desfecho do estudo foi a hemoglobina glicada (HbAlc), categorizada de acordo com seus índices em controlada (HbAlc entre 4\% a 6\%), moderada (HbAlc entre 6\% a 7\%) e mal controlada - HbAl maior que 7\% ${ }^{(13)}$.

Os dados foram obtidos por meio da aplicação de dois questionários traduzidos e validados para a língua portuguesa; o Questionário do Suporte Social (SSQ) e o Questionário de Atividades do Autocuidado em Diabetes - $\mathrm{QAD}^{(14)}$. Também foi utilizado um instrumento estruturado para se obter os dados sociodemográficos, antropométricos e clínicos.
O questionário do autocuidado com o diabetes foi desenvolvido para avaliar, de maneira sistematizada, as atividades de autocuidado em DM, no qual questiona a realização de atividades pelos indivíduos nos últimos sete dias, através da mensuração de cinco dimensões da terapêutica em DM: alimentação geral, alimentação específica, atividade física, uso de medicação e monitorização da glicemia, cuidados com os pés e hábito de fumar. A contabilização do escore é obtida para cada uma das dimensões por meio da média do número de dias (escala de 0-7), sendo zero a situação menos desejável e sete a mais favorável. Na dimensão dieta específica, os itens que questionam sobre o consumo de alimentos ricos em gordura e açúcar tiveram a pontuação do escore invertida, isto é, $7=0 ; 6=1 ;[\ldots]^{(14)}$.

$\mathrm{O}$ questionário do suporte social fornece dados de dimensões do suporte social como a presença/existência de indivíduos capazes de fornecer apoio em diferentes situações, a percepção/identificação de apoio disponível e a avaliação sobre a situação de suporte ${ }^{(15)}$. O SSQ é composto por 27 questões e cada questão solicita uma resposta em duas partes: na primeira, deve ser indicado o número de fontes de suporte social percebido (SSQ-N), podendo o respondente listar até nove possibilidades (ou a opção nenhum); na segunda parte, o respondente deve informar sobre essa satisfação com esse suporte, em uma escala do tipo Likert de 6 (seis) pontos (que varia de muito satisfeito a muito insatisfeito). Ambos os questionários foram validados para a população brasileira ${ }^{(14,15)}$.

Após aceitarem participar do estudo e assinarem o Termo de Consentimento Livre e Esclarecido, os participantes foram entrevistados individualmente, em ambiente privativo com aplicação de um questionário estruturado, contendo questões relacionadas aos dados sociodemográficos (idade, sexo, cor, religião, situação conjugal, renda, escolaridade), antropométricos (peso, altura, circunferência abdominal), hábitos de vida (uso de álcool, tabaco), além da busca no 
prontuário sobre comorbidades e do valor da hemoglobina glicada nos últimos três meses anteriores à entrevista. Neste momento, também foi investigado o autocuidado e o grau de satisfação com o suporte recebido através da aplicação dos questionários sobre o autocuidado em diabetes e o de suporte social, ambos já validados.

Os dados foram tabulados no Microsoft Excel ${ }^{\circ}$, versão 6.0 e analisados no software estatístico SAS versão 9.4 , sendo as análises descritivas dos dados representadas por frequências e as inferenciais por medidas de tendência central (média e mediana) e de variabilidade (desvio-padrão e amplitude). Para analisar as associações entre as variáveis qualitativas, foi aplicado o teste Qui-quadrado. Para todas as análises, o teste foi considerado significativo se o valor de p-alfa era igual ou inferior a 0,05 .

Quanto aos aspectos éticos, foram seguidas as diretrizes preconizadas para pesquisas com seres humanos pela Resolução n. ${ }^{\circ}$ 466/2012 do Conselho de
Nacional de Saúde e a coleta de dados somente ocorreu após aprovação do projeto pelo Comitê de Ética em Pesquisa da Unicamp, sob o número $716.105 / 2014$.

\section{RESULTADOS}

Dos 154 participantes, a maioria era do sexo feminino (62,34\%), bran$\cos (50 \%)$, que seguiam alguma religião $(93,5 \%)$ e aposentados $(61,6 \%)$. Quanto à situação conjugal e arranjo familiar, eram casados $(46,7 \%)$ e viviam sozinhos (26,6\%), a média do número de filhos foi 2,32 . No que diz respeito à idade, esta variou de 35 a 91 anos, apresentando uma média de 63,7 (DP $\pm 11,4)$. Em relação aos dados antropométricos e clínicos, a obesidade ficou evidente em $51,9 \%$ da amostra. O IMC apresentou uma média de $29,4 \mathrm{~kg} / \mathrm{m} 2$, o uso do tabaco e do álcool foram relatados por $5,8 \%$ e $12,3 \%$ da amostra, respectivamente. O tempo de tratamento variou de poucos meses a 32 anos, tendo uma média de 10,6 anos $(\mathrm{DP} \pm 7,3)$. A HbAlc variou de 4,7 a
$15 \%$, apresentando uma média de 7,7 \% e constatou se que $1,95 \%$ dos participantes apresentava controle glicêmico, além de uma média de glicemia de jejum igual a $145,4 \mathrm{mg} / \mathrm{dL}$ (DP $\pm 69,7$ ).

No que tange à média das atividades do autocuidado e do suporte social, as variáveis relacionadas ao consumo de frutas e ou vegetais $(6,0)$, o hábito de secar os espaços interdigitais $(6,2)$ e o tomar os hipoglicemiantes $(6,5)$ atingiram média próxima da pontuação máxima que é sete, mas a prática de atividade física $(2,7)$ obteve média abaixo do desejado, que é sete também. Em relação ao número de pessoas reconhecidas como as que oferecem apoio pelos diabéticos entrevistados, a média foi 1,2 pessoas por diabético com uma mediana de 1,15 e o grau de satisfação com o apoio social recebido teve uma média de 5,2 e mediana de 5,6 (Tabela 1 ).

A Tabela 2 mostra as associaçóes realizadas entre o controle glicêmico, as comorbidades encontradas neste estudo e o uso da insulina. Ficou evidente que os in-

Tabela 1. Atividades do autocuidado e percepção do suporte social dos pacientes diabéticos tipo 2. Juiz de Fora, MG, Brasil, $2017(n=154)$

$\begin{array}{lcccccccc} & \mathrm{n} & \text { Média } & \mathrm{DP} & \text { Mín. } & \text { Q1 } & \text { Media. } & \text { Q3 } & \text { Máx. } \\ \text { Seguiu dieta saudável } & 154 & 5,14 & 2,43 & 0 & 3 & 7,0 & 7 & 7 \\ \text { Seguiu orientação alimentar profissional } & 154 & 5,19 & 2,35 & 0 & 5 & 6,0 & 7 & 7 \\ \text { Comeu frutas e ou vegetais } & 154 & 6,01 & 1,68 & 0 & 5 & 7,0 & 7 & 7 \\ \text { Comeu alimentos gordurosos } & 154 & 3,73 & 2,67 & 0 & 1 & 3,0 & 7 & 7 \\ \text { Comeu doce } & 154 & 1,97 & 2,43 & 0 & 0 & 1,0 & 3 & 7 \\ \text { Realizou atividade física 30 mim } & 154 & 2,95 & 2,93 & 0 & 0 & 2,5 & 6 & 7 \\ \text { Praticou atividade física específica } & 154 & 2,73 & 2,88 & 0 & 0 & 2,0 & 6 & 7 \\ \text { Avaliou a glicemia } & 154 & 4,34 & 2,96 & 0 & 1 & 6,0 & 7 & 7 \\ \text { Avaliou a glicemia conforme recomendação profissional } & 154 & 4,04 & 3,16 & 0 & 0 & 5,5 & 7 & 7 \\ \text { Examinou os pés } & 154 & 5,40 & 2,60 & 0 & 4 & 7,0 & 7 & 7 \\ \text { Examinou dentro dos sapatos } & 154 & 4,49 & 3,13 & 0 & 0 & 7,0 & 7 & 7 \\ \text { Secou os espaços interdigitais } & 154 & 6,21 & 2,01 & 0 & 7 & 7,0 & 7 & 7 \\ \text { Tomou os hipoglicemiantes (insulina e comprimidos) } & 154 & 6,53 & 1,55 & 0 & 7 & 7,0 & 7 & 7 \\ \text { Tomou insulina } & 154 & 4,49 & 3,29 & 0 & 0 & 7,0 & 7 & 7 \\ \text { Pessoas percebidas como promotoras de suporte social } & 154 & 1,21 & 0,46 & 0,19 & 1,00 & 1,15 & 1,48 & 2,63 \\ \text { Satisfação com o suporte social } & 154 & 5,21 & 1,10 & 1,22 & 4,89 & 5,69 & 6,00 & 6,00 \\ \text { Notas: DP - Desvio padrão; Mín. - Minimo; Media. - Mediana; Máx. -Măximo. } & & & & & & & \end{array}$




\section{artigo}

Santos, M.M.D.; Peters, A.A.; Figueiredo, M.A.G.; Carreiro, M.A.; Magalhães, M.C.; Araújo, E.P.;

Controle glicêmico, suporte social percebido e o autocuidado de indivíduos com diabetes tipo 2

divíduos com a HbAlc classificada como mal controlada possuíam hipertensão $(\mathrm{p}=0,0136)$, obesidade $(\mathrm{p}=0,0042)$, nefropatias $(p=0,0207)$, depressão $(p$ $=0,0092)$ e faziam uso de insulina ( $\mathrm{p}=$ $0,0030)$, demonstrando associação esta- tisticamente significante entre elas.

Ao se associar as atividades de autocuidado dos pacientes com DM2 e o suporte social (SS) recebido, não houve associação estatística significativa.

\section{DISCUSSÃO}

Neste estudo, a maioria dos participantes com DM2 era do sexo feminino, apresentaram uma média de idade de 63,7 anos, baixa escolaridade e renda,

Tabela 2. Associação das comorbidades dos pacientes diabéticos tipo 2 com a classificação da HbA1c. Juiz de Fora, MG,

Brasil, 2017

HbA1c classificação

\begin{tabular}{|c|c|c|c|}
\hline Variável & $\begin{array}{c}\text { Controlada/moderada } \\
4 \% \text { a } 7 \% \text { - n (\%) }\end{array}$ & $\begin{array}{l}\text { Mal controlada } \\
>7 \%-\mathrm{n}(\%)\end{array}$ & p-valor* \\
\hline Hipertensão & & & 0,0136 \\
\hline Não & $17(65,38)$ & $9(34,62)$ & \\
\hline Sim & $50(39,06)$ & $78(60,94)$ & \\
\hline Obesidade & & & 0,0042 \\
\hline Não & $41(55,41)$ & $33(44,59)$ & \\
\hline Sim & $26(32,50)$ & $54(67,50)$ & \\
\hline Nefropatias & & & 0,0207 \\
\hline Não & $65(46,43)$ & $75(53,57)$ & \\
\hline Sim & $2(14,29)$ & $12(85,71)$ & \\
\hline Retinopatias & & & 0,1737 \\
\hline Não & $42(48,28)$ & $45(51,72)$ & \\
\hline Sim & $25(37,31)$ & $42(62,69)$ & \\
\hline Neuropatias & & & 0,0607 \\
\hline Não & $29(53,7)$ & $25(46,30)$ & \\
\hline Sim & $38(38,00)$ & $62(62,00)$ & \\
\hline Hipotireoidismo & & & 0,8852 \\
\hline Não & $58(43,28)$ & $76(56,72)$ & \\
\hline Sim & $9(45,00)$ & $11(55,00)$ & \\
\hline Depressão & & & 0,0092 \\
\hline Não & $64(47,41)$ & $71(52,59)$ & \\
\hline Sim & $3(15,79)$ & $16(84,21)$ & \\
\hline Doenças articulares e musculares & & & 0,1476 \\
\hline Não & $30(50,85)$ & $29(49,15)$ & \\
\hline $\operatorname{Sim}$ & $37(38,95)$ & $58(61,05)$ & \\
\hline Dislipidemia & & & 0,4475 \\
\hline Não & $19(48,72)$ & $20(51,28)$ & \\
\hline Sim & $48(41,74)$ & $67(58,26)$ & \\
\hline Insulina & & & 0,0030 \\
\hline Não & $35(58,33)$ & $25(41,67)$ & \\
\hline Sim & $32(34,04)$ & $62(65,96)$ & \\
\hline
\end{tabular}


aspectos parecidos com outras pesquisas $^{(16,17)}$. Vários estudos nacionais e internacionais têm evidenciado a preponderância de DM tipo $2 \mathrm{em}$ pessoas da faixa etária compreendida entre 45 e 64 anos, aumentando significativamente em indivíduos de 65 anos de idade ou mais ${ }^{(2,18)}$, reforçando ser este um problema de saúde pública frente à expectativa de vida aumentada do povo brasileiro, favorecendo o envelhecimento e, consequentemente, maior prevalência de doenças crônicas não transmissíveis, como o $\mathrm{DM}^{(2,19)}$.

Tal fato corrobora com os estudos que afirmam que a idade é um fator dificultador para o alcance de um bom controle glicêmico, devido, principalmente, às dificuldades de entendimento das informações sobre a doença e aplicação destas orientações no autocuidado, decorrentes do déficit cognitivo e funcional progressivo com o decorrer dos anos, ou aumento do grau de dependência para efetuar as ações ${ }^{(17,20)}$.

$\mathrm{Na}$ avaliação das medidas antropométricas e clínicas, a obesidade e a cintura abdominal estiveram com níveis elevados, de acordo com a literatura ${ }^{(4,21)}$.

Os agravos à saúde apresentados nesta pesquisa, como a Hipertensão Arterial Sistêmica (HAS), cardiopatias, doenças osteomusculares, neuropatia e a retinopatia, são comorbidades citadas em vários estudos ${ }^{(22,23)}$. Isto se justifica principalmente pela intensa relação destas com o DM, além da síndrome metabólica, que geralmente precede o DM e é responsável em grande parte pela HAS que aumenta os efeitos negativos da falta de controle glicêmico, além do desenvolvimento de cardiopatias e de doença aterosclerótica $^{(22)}$.

As doenças cardiovasculares (DCVs) são as principais causas de morte na população brasileira, responsáveis por $20 \%$ das mortes em nossa população com mais de 30 anos de idade ${ }^{(24)}$. Neste estudo, as mais prevalentes foram o infarto agudo do miocárdio (IAM) e o acidente vascular cerebral (AVC).

O bom controle glicêmico poderá minimizar ou controlar as complicações do diabetes. O parâmetro essencial na avaliação do controle do DM é a dosagem da HbA1C. Estudos destacam a íntima relação entre o controle glicêmico preciso, evidenciado pela $\mathrm{HbA} 1 \mathrm{C}$ e a prevenção do aparecimento ou evolução das complicações crônicas do DM como a neuropatia e retinopatia diabética, doenças cardiovasculares, dentre outras ${ }^{(23,25)}$.

Os resultados do estudo UKPDS ${ }^{(24)}$ apontaram que as complicações microvasculares do DM2 são diminuídas quando há um bom controle glicêmico, o que foi visto no referido estudo quando houve tratamento intensivo dos diabéticos e obtenção da média da HbA1C igual a 7, reduzindo os riscos em $25 \%$.

O alcance do bom controle glicêmico foi pequeno entre os diabéticos tipo 2. Tal fato pode sofrer a influência da idade, do grau de escolaridade, condições socioeconômicas e da complexidade do tratamento aliada à ausência de um bom acompanhamento e controle desta patologia ${ }^{(26)}$.

A HAS é a principal comorbidade associada ao DM e, nas unidades básicas de saúde, a hipertensão é atendida de uma maneira especial através do Programa de Hipertensão Arterial com ações de promoção à saúde, dispensação de medicamentos hipotensores, diuréticos, o que de certa forma, diminui os riscos de complicações cardiovasculares e causa impacto no controle metabólico ${ }^{(27)}$.

A obesidade, problema de saúde pública em todo mundo, demonstra a não aderência ao plano alimentar, e é um importante fator de risco também para HAS, doenças cardiovasculares, e para o próprio DM2, principalmente quando associada à presença excessiva do tecido adiposo visceral ${ }^{(20)}$, pois os indivíduos com maior quantidade de tecido adiposo tendem a ter resistência à insulina. Caracteriza também a tendência da obesidade de dificultar o alcance de um bom controle glicêmico, podendo aumentar os problemas metabólicos e cardiovasculares pré-existentes ${ }^{(4,20)}$.

A depressão foi uma das comorbidades também em evidência neste estudo, gerando preocupação com seu efeito deletério no tratamento do diabetes, visto que os sintomas depressivos podem prejudicar a manutenção do tratamento, principalmente no que diz respeito à dieta e à atividade física. Isto poderá agravar o controle glicêmico e aumentar o risco de complicações, além de estimular o maior índice de tabagismo e aumento da morbidade e mortalidade, decorrentes da menor falta de manutenção do tratamento dietético e medicamentoso ${ }^{(28,29)}$.

A nefropatia diabética (ND) também foi identificada como uma complicação importante. Considera-se o DM como a causa mais relevante da doença renal, sendo o tipo 2 o de maior incidência e prevalência na população brasileira e mundial e responsável pela maior parte das pessoas inscritas nos programas de diálise ${ }^{(30)}$. Esta complicação microvascular também é decorrente das alterações fisiológicas ocasionadas pela hiperglicemia persistente, aliada à predisposição genética e tem como marcador a microalbuminúria, importante também para a avaliação cardiovascular, haja vista a associação que ocorre entre a proteinúria e a doença cardiovascular, responsável por um alto índice de mortalidade dentre estas pessoas ${ }^{(31)}$.

A pontuação máxima atingida em vários parâmetros da vida, como alimentação adequada e hábitos de higiene, demonstra que há uma preocupação com um dos pilares do tratamento do DM, pois a alimentação saudável é um elemento importantíssimo do tratamento com vistas ao controle da doençą ${ }^{(4)}$.

Outro item que ficou em evidência pela proximidade de sua média com a ideal foi o hábito de secar os espaços interdigitais. Este é um cuidado que os portadores de diabetes devem ter diariamente, visando à integridade de sua pele e, consequentemente, a prevenção da formação de feridas infectadas nos pés e culminar com futuras amputações ${ }^{(4)}$.

Neste estudo, não foi feita a categorização da variável sexo relacionada ao cuidado com os pés, ficando esta ação bem pontuada para ambos os sexos, po- 


\section{artigo}

Santos, M.M.D.; Peters, A.A.; Figueiredo, M.A.G.; Carreiro, M.A.; Magalhães, M.C.; Araújo, E.P.;

Controle glicêmico, suporte social percebido e o autocuidado de indivíduos com diabetes tipo 2

rém, é fato que os homens apresentam maiores dificuldades quando comparados com as mulheres neste cuidado. Um estudo prospectivo ${ }^{(32)}$, seguido por sete anos, identificou o sexo masculino como um fator de risco para amputação em pacientes com pé diabético, aliado a outros fatores juntamente como o longo tempo de diagnóstico, hemoglobina glicada elevada, retinopatia e o uso da insulina no tratamento.

Os entrevistados se mostraram bastante satisfeitos com o suporte social recebido, porém esses mesmos indivíduos que disseram estar satisfeitos não apresentaram um controle glicêmico efetivo. $\mathrm{O}$ apoio de familiares tem sido compreendido como um recurso facilitador para os diabéticos que lidam com o estresse da doença e para a qualidade de vida ${ }^{(33)}$. Um estudo finlandês desenvolvido em indivíduos diabéticos concluiu que o suporte social mais elevado possibilita melhor qualidade de vida ${ }^{(34)}$.

As limitações deste estudo referem-se ao delineamento transversal que não permite situar a relação de causa e efeito, porém permitiu associações estatísticas significantes.

\section{CONCLUSÃO}

Os resultados alcançados neste estudo demonstraram que não houve associação entre o controle glicêmico, as variáveis sociodemográficas e clínicas e o suporte social. Em relação às comorbidades, variáveis estas, associadas com o controle glicêmico, a HAS, obesidade, AVC e depressão, apresentaram associação estatística significativa com a $\mathrm{HbAlc}$ mal controlada.

Estas patologias podem levar o diabético ao descontrole glicêmico devido às implicações metabólicas que ocasionam, além de dificultarem o paciente no desempenho correto das etapas do tratamento do diabetes, como a prática de atividade física e seguir a dieta prescrita.

A implementação de estratégias educativas por parte dos profissionais de saúde, que possibilite aos diabéticos a adoção de práticas de saúde com atitudes necessárias para o enfrentamento das demandas do tratamento e cuidados com o diabetes, visando o alcance de um bom controle glicêmico, pois em muitos diagnósticos, a complicação já está instalada.

Desta forma, as atividades de educação em saúde desenvolvidas pelos profissionais enfermeiros nas unidades de saúde podem ser um bom momento para proporcionar aos diabéticos a convivência com outras pessoas que tiveram boas experiências com o emprego de ações em prol de seu tratamento e, assim, favorecer na busca por um bom controle glicêmico.

Em nosso trabalho, o suporte social declarado pelos indivíduos como satisfatório, parece não ser o adequado para o seu tratamento e cuidado com a saúde.

O controle glicêmico adequado é um desafio para as equipes de saúde e para os pacientes e necessita de várias formas de enfrentamento e os pacientes precisam ser empoderados para o cuidado à sua saúde. O paciente que apresenta maior independência na realização dos cuidados relacionados à sua saúde pode apresentar um controle melhor dos parâmetros relacionados à sua doença.

\section{REFERÊNCIAS}

1. International Diabetes Federation. IDF Diabetes atlas [Internet]. 7th ed. Brussels, Belgium: International Diabetes Federation; 2015 [cited 2017 May, 27]. Available from: http://www. diabetesatlas.org

2. Malerbi D, Franco L. Multicenter study of the prevalence of diabetes mellitus and impaired glucose tolerance in the urban Brazilian population aged 30-69 yr. The Brazilian Cooperative Group on the Study of Diabetes Prevalence. Diabetes Care. 1992;15(11):1509-16. doi: 10.2337/diacare.15.11.1509

3. Bosi P, Carvalho A, Contrera D, Casale G, Pereira M, Gronner $\mathrm{M}$, et al. Prevalence of diabetes and impaired glucose tolerance in the urban population of 30 to 79 years of the city of São Carlos, São Paulo. Arq Bras Endocrinol Metabol. 2009;53(6):726-32. doi: 10.1590/S0004-27302009000600006

4. Sociedade Brasileira de Diabetes. Diretrizes da Sociedade Brasileira de Diabetes: 2014-2015 [Internet]. São Paulo: AC Farmacêutica; 2015 [cited 2019 May, 27]. Available from: http://www. diabetes.org.br/publico/images/2015/area-restrita/diretrizes-sbd-2015.pdf

5. Schmidt MI, Hoffmann JF, Diniz M, Lotufo PA, Griep RH, Bensenor IM, et al. High prevalence of diabetes and intermediate hyperglycemia - The Brazilian Longitudinal Study of Adult Health (ELSA-Brasil). Diabetol Metab Syndr. 2014;6(1):123. doi: 10.1186/1758-5996-6-123

6. Villas-Boas LG, Santos C, Foss-Freitas M, Pace A. A relação entre o apoio social e as características sociodemográficas das pessoas com diabetes mellitus. Rev Gaúcha Enferm. [Internet]. 2009 [cited 2017 May, 27];30(3):390-6. Available from: http:// www.seer.ufrgs.br/index.php/RevistaGauchadeEnfermagem/ article/view/8635/6991

7. Barrera M. Distinctions between social support concepts, measures, and models. Am J Community Psychol. 1986;14(4):41345. doi: 10.1007/BF00922627

8. Pedro ICS, Rocha SMM, Nascimento LC. Social support and social network in family nursing: reviewing concepts. Rev Latino-Am Enfermagem. 2008;16(2):324-7. doi: 10.1590/s010411692008000200024

9. Sociedade Brasileira de Diabetes. Diretrizes da Sociedade Brasileira de Diabetes [Internet]. Itapevi: A. Araújo Silva Farmacêutica; 2009 [cited 2017 May 27]. Available from: http://dms.ufpel. edu.br/ares/bitstream/handle/123456789/270/11\%20\%20\%20 2009\%20diretrizes\%20diabete.pdf?sequence $=1$ 


\section{REFERÊNCIAS}

10. Rodrigues F, Zanetti M, Santos M, Martins T, Sousa V, Teixeira C. Knowledge and attitude: important components in diabetes education. Rev Latino-Am Enfermagem. 2009;17(4):468-73. doi: 10.1590/s0104-11692009000400006

11. Sherbourne CD, Stewart AL. The MOS social support survey. Soc Sci Med. 1991;32(6):705-14. doi: 10.1016/02779536(91)90150-B

12. Shumaker S, Brownell A. Toward a Theory of Social Support: closing conceptual gaps. J Soc Issues. 1984;40(4):11-36. doi: 10.1111/j.1540-4560.1984.tb01105.x

13. Sociedade Brasileira de Diabetes. Diretrizes da Sociedade Brasileira de Diabetes: 2013-2014 [Internet]. São Paulo: AC Farmacêutica; 2014 [cited 2017 Apr, 7]. Available from: http://www. diabetes.org.br/publico/images/pdf/diretrizes-sbd.pdf

14. Michels M, Coral M, Sakae T, Damas T, Furlanetto L. Questionnaire of Diabetes Self-Care Activities: translation, cross-cultural adaptation and evaluation of psychometric properties. Arq Bras Endocrinol Metabol. 2010;54(7):644-51. doi: 10.1590/ S0004-27302010000700009

15. Matsukura T, Marturano E, Oishi J. O Questionário de Suporte Social (SSQ): estudos da adaptação para o português. Rev Latino-Am Enfermagem. 2002;10(5):675-81. doi: 10.1590/S010411692002000500008

16. Freitas EF, Moreira OC, Oliveira CEP, Doimo LA, Loch MR, Freitas EF, et al. Prevalence of diabetes mellitus and exercise practice in individuals who sought care at the family health strategy of Viçosa/MG. Rev Educ Física UEM. 2015;26(4):549-56. doi: 10.4025/reveducfis.v26i4.25202

17. Lira Neto J, Silva AP, Araújo MFM, Damasceno MMC, Lan$\operatorname{dim}$ MBP, Freitas RWJF, et al. Metabolic control and medication adherence in people with diabetes mellitus. Acta Paul Enferm. 2017;30(2):152-8. doi: 10.1590/1982-0194201700024

18. Boyle J, Thompson T, Gregg E, Barker L, Williamson D. Projection of the year 2050 burden of diabetes in the US adult population: dynamic modeling of incidence, mortality, and prediabetes prevalence. Popul Health Metr 2010;8(1):29. doi: 10.1186/1478-7954-8-29

19. Souza L, Chalita F, Reis A, Teixeira C, Gicovate Neto C, Bastos $D$, et al. Prevalence of diabetes mellitus and risk factors in Campos dos Goytacazes, RJ. Arq Bras Endocrinol Metabol. 2003;47(1):69-74. doi: 10.1590/S0004-27302003000100011

20. Faria HTG, Rodrigues FFL, Zanetti ML, Araújo MFM, Damasceno MMC. Factors associated with adherence to treatment of patients with diabetes mellitus. Acta Paul Enferm. 2013;26(3):231-7. doi: 10.1590/S0103-21002013000300005

21. Villas-Boas LG, Lima MLSAP, Pace AE. Adherence to treatment for diabetes mellitus: validation of instruments for oral antidiabetics and insulin. Rev Latino-Am Enfermagem. 2014;22(1):11-8. doi: 10.1590/0104-1169.3155.238

22. Gus I, Fischmann A, Medina C. Prevalence of risk factors for coronary artery disease in the Brazilian State of Rio Grande do
Sul. Arq Bras Cardiol. 2002;78(5):484-90. doi: 10.1590/S0066$782 \times 2002000500005$

23. U.S. Department of Health and Human Services. DCCT and EDIC. The Diabetes Control and Complications Trial and Follow-up Study [Internet]. 1983 [cited 2019 Feb, 17]. Available from: https://www.niddk.nih.gov/about-niddk/research-areas/diabetes/dcct-edic-diabetes-control-complications-trial-follow-up-study/Documents/DCCT-EDIC_508.pdf

24. Mansur A, Favarato D. Trends in mortality rate from cardiovascular disease in Brazil, 1980-2012. Arq Bras Cardiol. 2016;107(1):20-5. doi: 10.5935/abc.20160077

25. King P, Peacock I, Donnelly R. The UK Prospective Diabetes Study (UKPDS): clinical and therapeutic implications for type 2 diabetes. Br J Clin Pharmacol. 1999;48(5):643-8. doi: 10.1046/j. 1365-2125.1999.00092.x

26. Lima ACS, Araújo MFM, Freitas RWJF, Zanetti ML, Almeida PC, Damasceno MMC, et al. Risk factors for Type 2 Diabetes Mellitus in college students: association with sociodemographic variables. Rev Latino-Am Enfermagem. 2014;22(3):484-90. doi: 10.1590/0104-1169.3053.2441

27. Gomides DS, Villas-Boas LCG, Coelho ACM, Pace AE. Self-care of people with diabetes mellitus who have lower limb complications. Acta Paul Enferm. 2013;26(3):289-93. doi: 10.1590/ S0103-21002013000300014

28. Moreira R, Papelbaum M, Appolinario J, Matos A, Coutinho W, Meirelles R, et al. Diabetes mellitus and depression: a systematic review. Arq Bras Endocrinol Metabol. 2003;47(1):19-29. doi: 10.1590/S0004-27302003000100005

29. Leite P. Depressão e diabetes mellitus. In: Sociedade Brasileira de Diabetes. Diabetes na prática clínica [Internet]. São Paulo: Sociedade Brasileira de Diabetes; 2014 [cited 2019 Feb, 20]. Available from: http://www.diabetes.org.br/ebook/component/ k2/item/48-depressao-e-diabetes-mellitus

30. Murussi M, Murussi N, Campagnolo N, Silveiro S. Early detection of diabetic nephropathy. Arq Bras Endocrinol Metabol. 2008;52(3):442-51. doi: 10.1590/S0004-27302008000300004

31. Sampaio E, Almeida H, Delfino V. Nephropathy and retinopathy in type 1 diabetics assisted by a universitary multiprofessional program. Arq Bras Endocrinol Metabol. 2007;51(3):410-8. doi: 10.1590/S0004-27302007000300008

32. Smanioto FN, Haddad MCFL, Rossaneis MA. Self-care into the risk factors in diabetic foot ulceration: cross-sectional study. Online Braz J Nurs. 2014;13(3):343-52. doi: 10.5935/16764285.20144680

33. Nunes M. Apoio Social na Diabetes. Millenium [Internet]. 2005 [citado 2019 Ago 05]; 31(10):135-49. Available from: https://revistas.rcaap.pt/millenium/article/download/8423/6009

34. Aalto AM, Uutela A, Aro AR. Health related quality of life among insulin-dependent diabetics: disease-related and psychosocial correlates. Patient Educ Couns. 1997;30(3):215-25. doi: 10.1016/S0738-3991(96)00963-9 